\title{
Inpatient prescription charts on medical wards for older people - room for improvement?
}

\author{
Authors: Sarah Start, Soma Kar, Sohail Mughal and Balasubramanian Venkatesh
}

\section{Aims}

The aim of the study was to determine adherence to MidYorkshire Hospitals NHS Trust inpatient prescribing standards while implementing change and education in written prescribing to promote safe prescribing and patient safety.

\section{Methods}

The retrospective audit was undertaken at Pinderfields General Hospital in a medical ward for older people with use of a generic audit prescribing proforma. This was used to determine compliance with correct patient identification, allergy status, senior review and venous thromboembolism (VTE) risk assessment and prescription. The initial audit cycle included a random sample of 10 current inpatients' prescription charts on the ward over a period of 5 days.

Results identified two key areas for improvement: senior review and VTE prophylaxis. We then implemented the promotion of an algorithm: Swabs, Thromboprophylaxis, Antibiotics, Cannula, Oxygen, Drug chart, and Do Not Attempt Cardiopulmonary Resuscitation status (STACODD) during daily ward rounds. We reviewed the compliance with a second audit cycle 4 months later. Data were obtained from the patient's current medical notes, which included the inpatient prescription chart, intravenous fluid chart and any chart that had previously been in use during the current admission prior to transcription. Once the data were collected, the results were compiled into Microsoft Excel and data analysis was performed.

\section{Results}

We demonstrated a $10 \%$ increase in the number of prescription charts that had been reviewed by a senior post-implementation of STACODD. Despite the percentage increase, the overall total of senior reviews remained low (20\%). Compliance with VTE prophylaxis prescribing was shown to have increased from an initial $10 \%$ on the first audit cycle to $50 \%$ on the second cycle. In the $50 \%$ that had been completed, all sections had been completed fully and the written prescription itself had been prescribed correctly.

\section{Conclusions}

The implementation of STACODD onto the older care ward had an improvement on the number of prescriptions that had been reviewed by a senior and had correct VTE prescriptions. The implementation achieved the aim of educating other healthcare professionals and promoted safe prescribing. The team, however, noted that the compliance could be increased further and allowed us to gather evidence in order to identify specific areas of prescribing that required improvement. The implication of this resulted in further education of healthcare professionals on the importance of good prescribing and enabled use of the algorithm, which can be used on a daily basis within ward rounds.

\section{Conflict of interest statement}

There are no conflicts of interest to declare. 